\title{
Isonormal surfaces: a new tool for the multidimensional dynamical analysis of iterative methods for solving nonlinear systems
}

\author{
Raudys Rafael Capdevila ${ }^{1}$, Alicia Cordero ${ }^{1}$, and Juan Ramon Torregrosa ${ }^{1}$ \\ ${ }^{1}$ Universidad Politécnica de Valencia
}

October 23,2020

\begin{abstract}
The dynamical behavior of the rational vectorial operator associated with a multidimensional iterative method in polynomial systems, gives us interesting information about the stability of the iterative scheme. The stability of fixed points, dynamic planes, bifurcation diagrams, etc. are known tools that act in this sense. In this manuscript, we introduce a new tool, which we call isonormal surface, to complement the information about the stability of the iterative method provided by the dynamic elements mentioned above. These dynamical instruments are used for analyze the stability of a parametric family of multidimensional iterative schemes in terms of the value of the parameter. Some numerical tests confirm the obtained dynamical results.
\end{abstract}

\section{Hosted file}

ArticleDynamicalAnalysis_v2.pdf available at https://authorea.com/users/369648/articles/ 488454-isonormal-surfaces-a-new-tool-for-the-multidimensional-dynamical-analysis-ofiterative-methods-for-solving-nonlinear-systems

\section{Hosted file}

ArticleDynamicalAnalysis_v2.tex available at https://authorea.com/users/369648/articles/ 488454-isonormal-surfaces-a-new-tool-for-the-multidimensional-dynamical-analysis-ofiterative-methods-for-solving-nonlinear-systems 

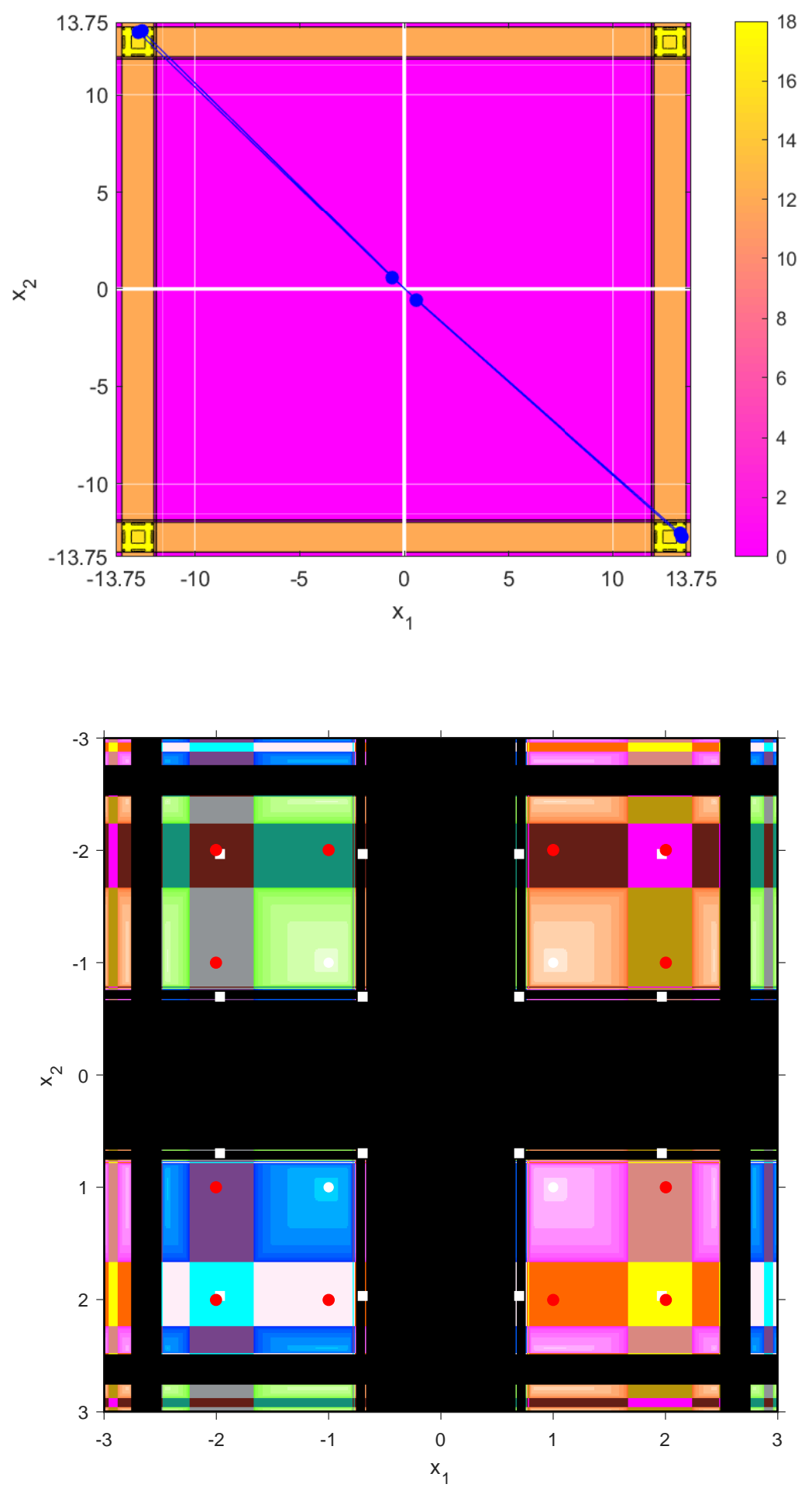

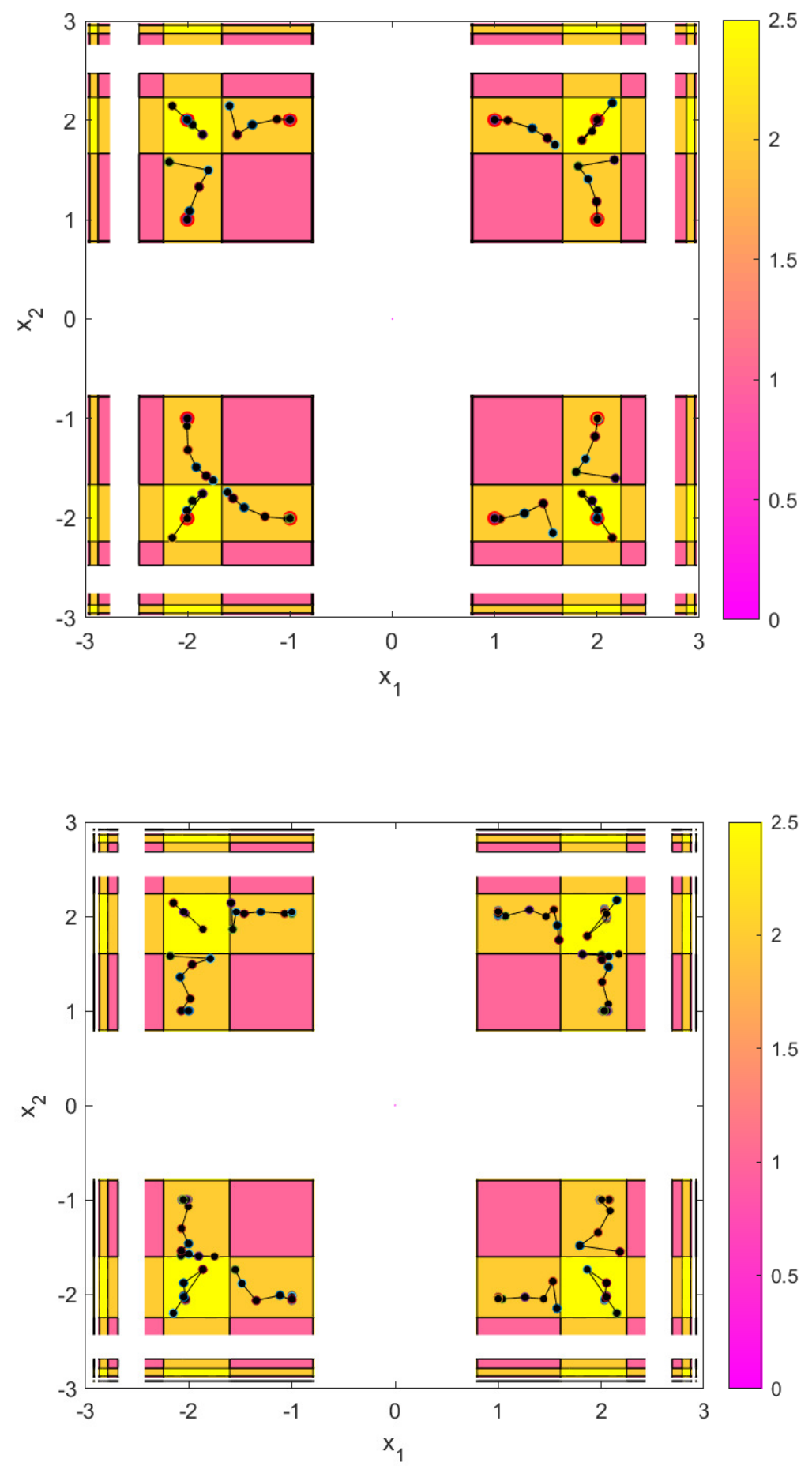

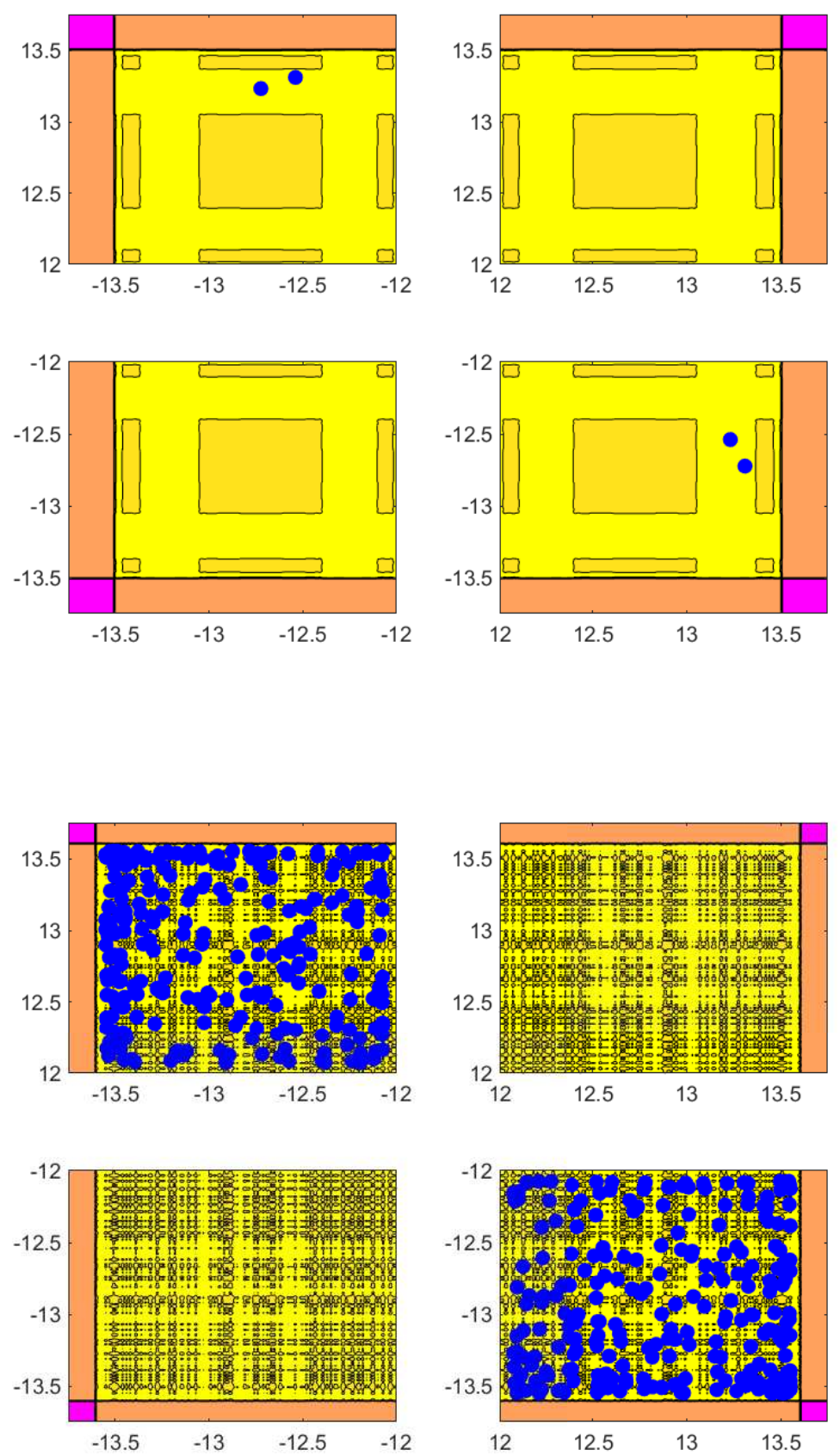

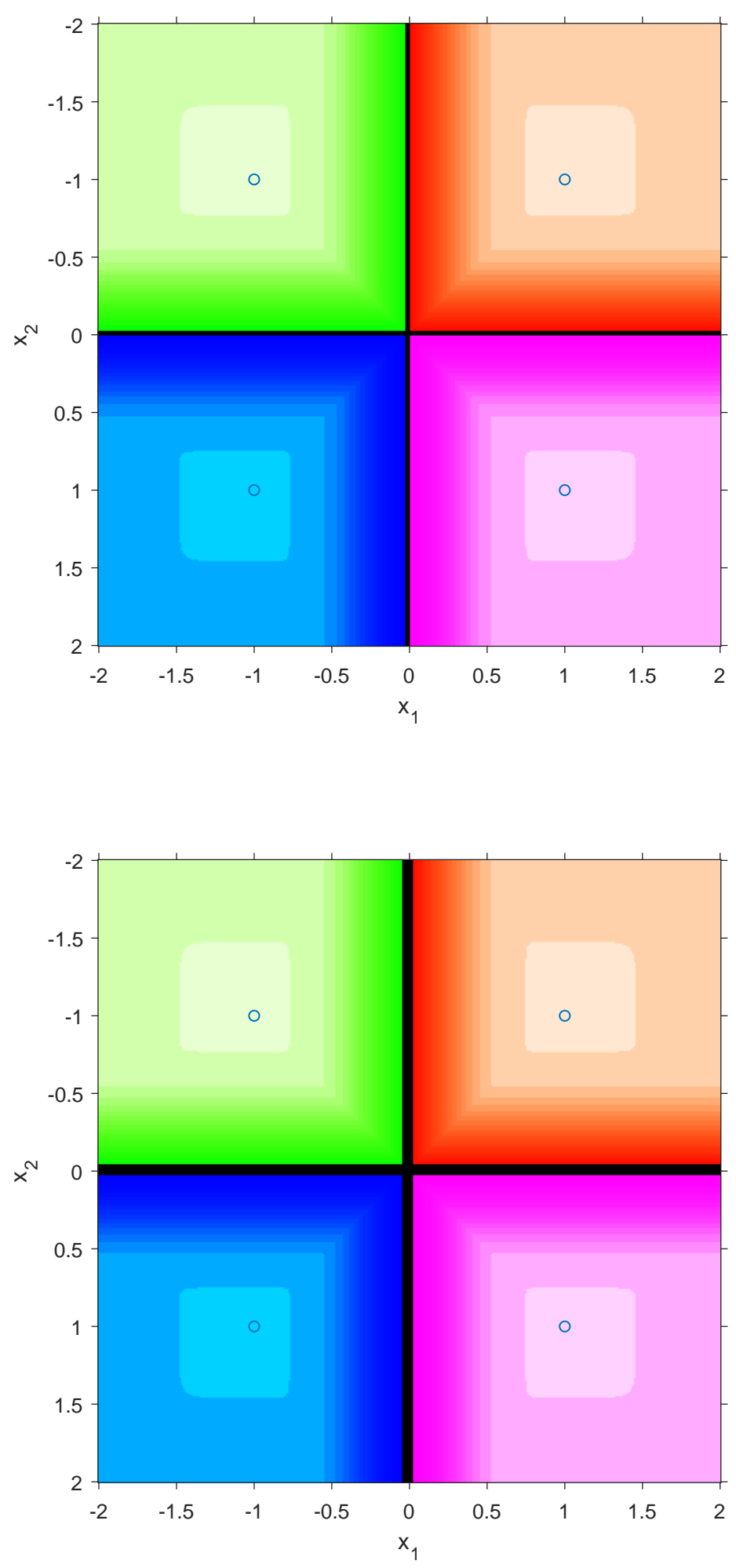

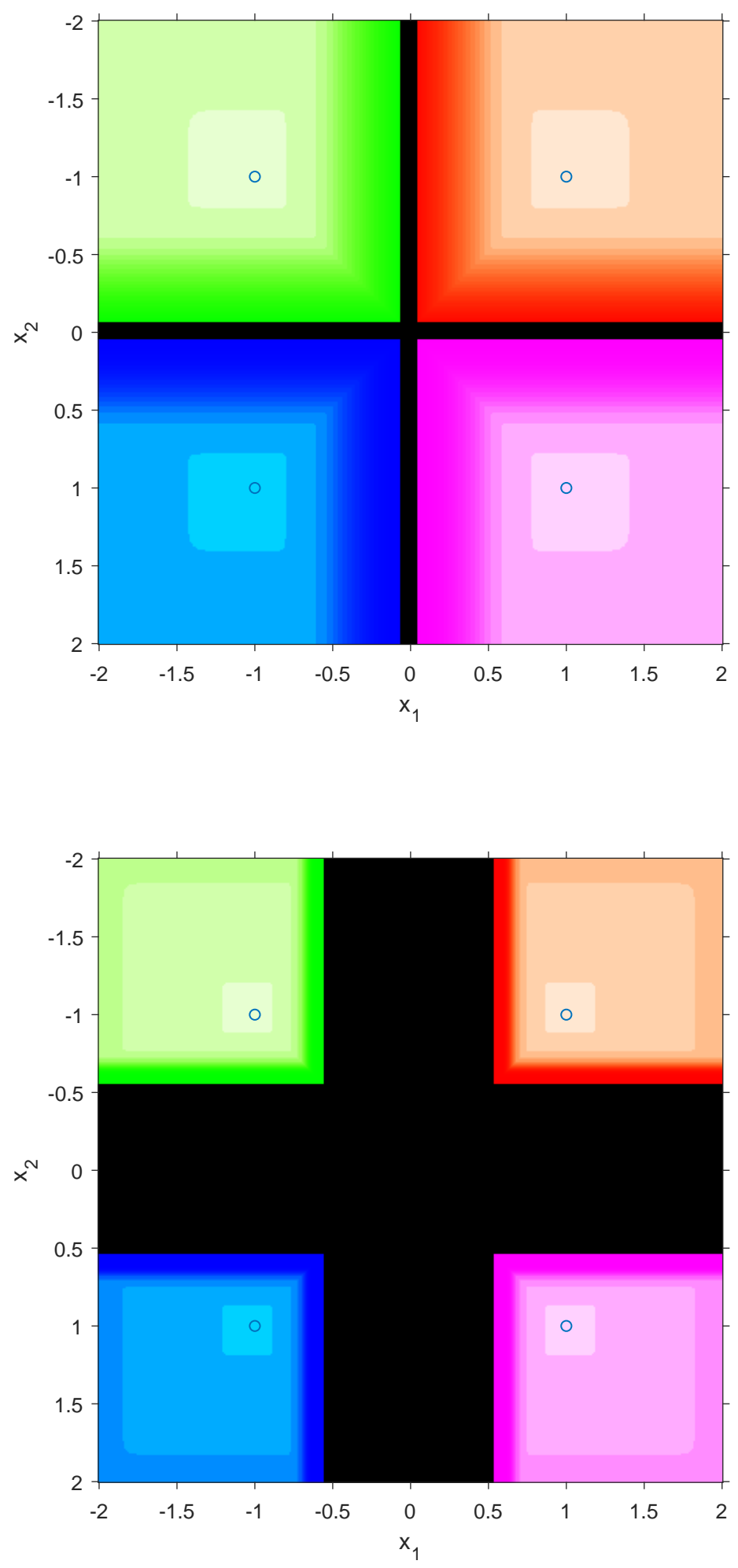

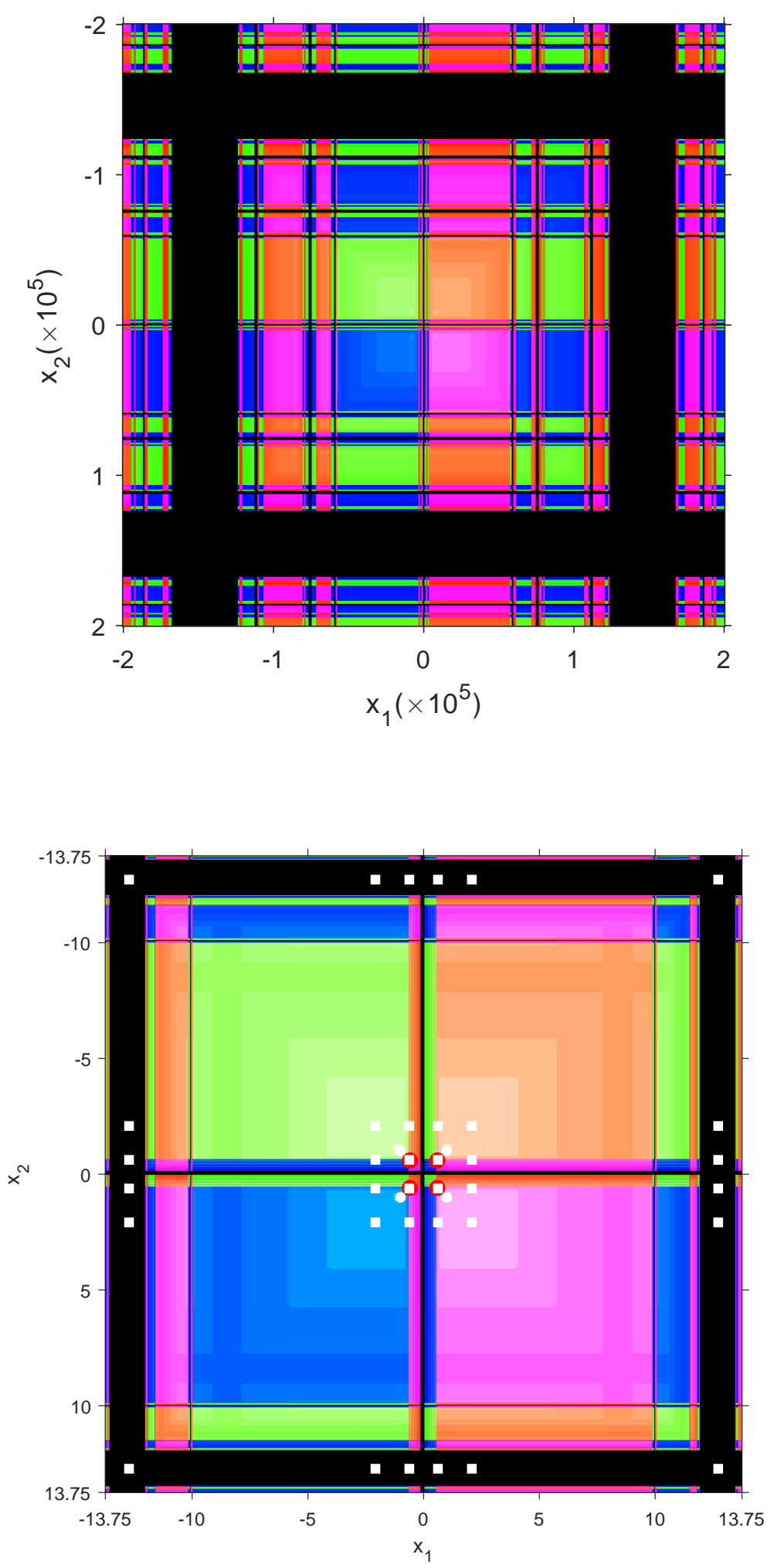

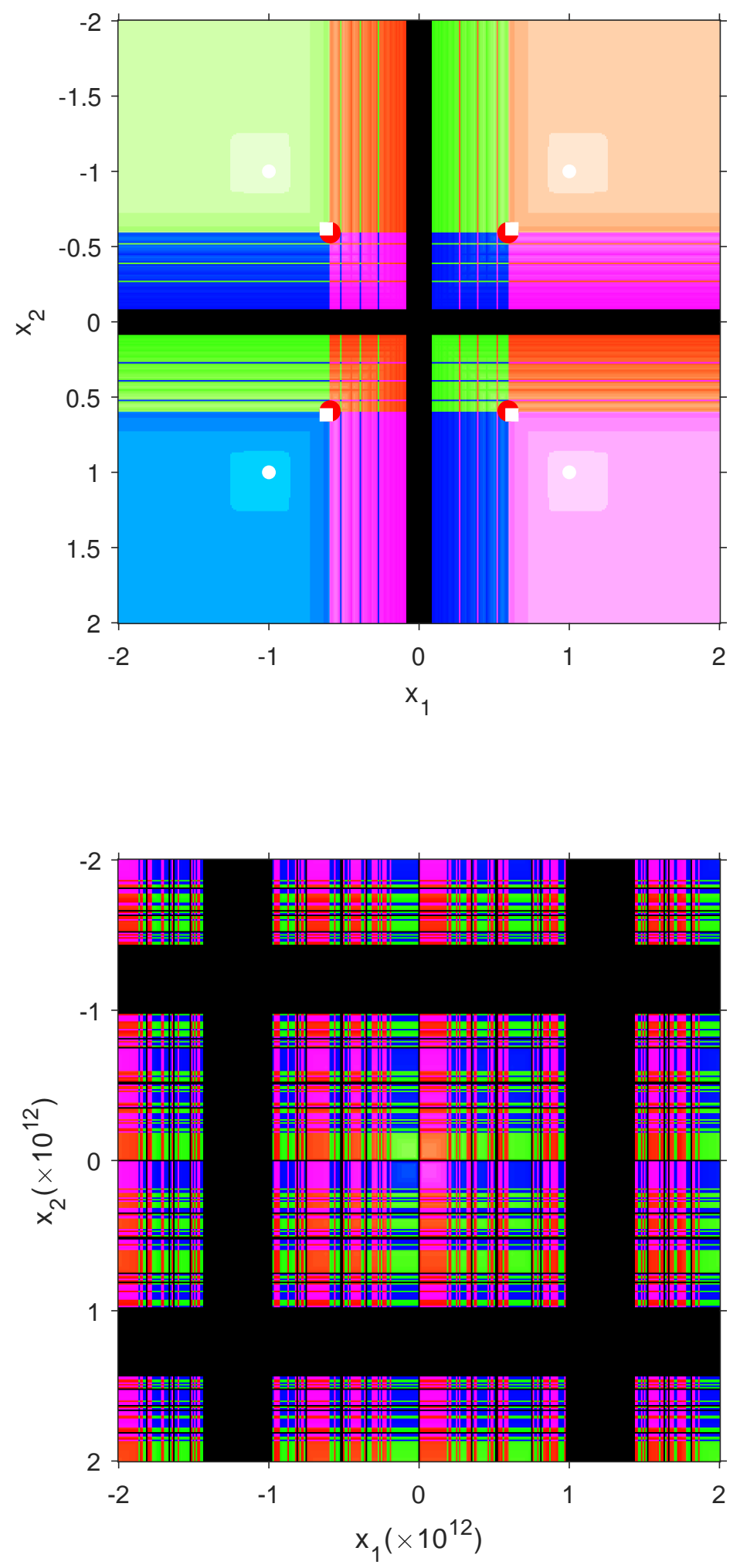

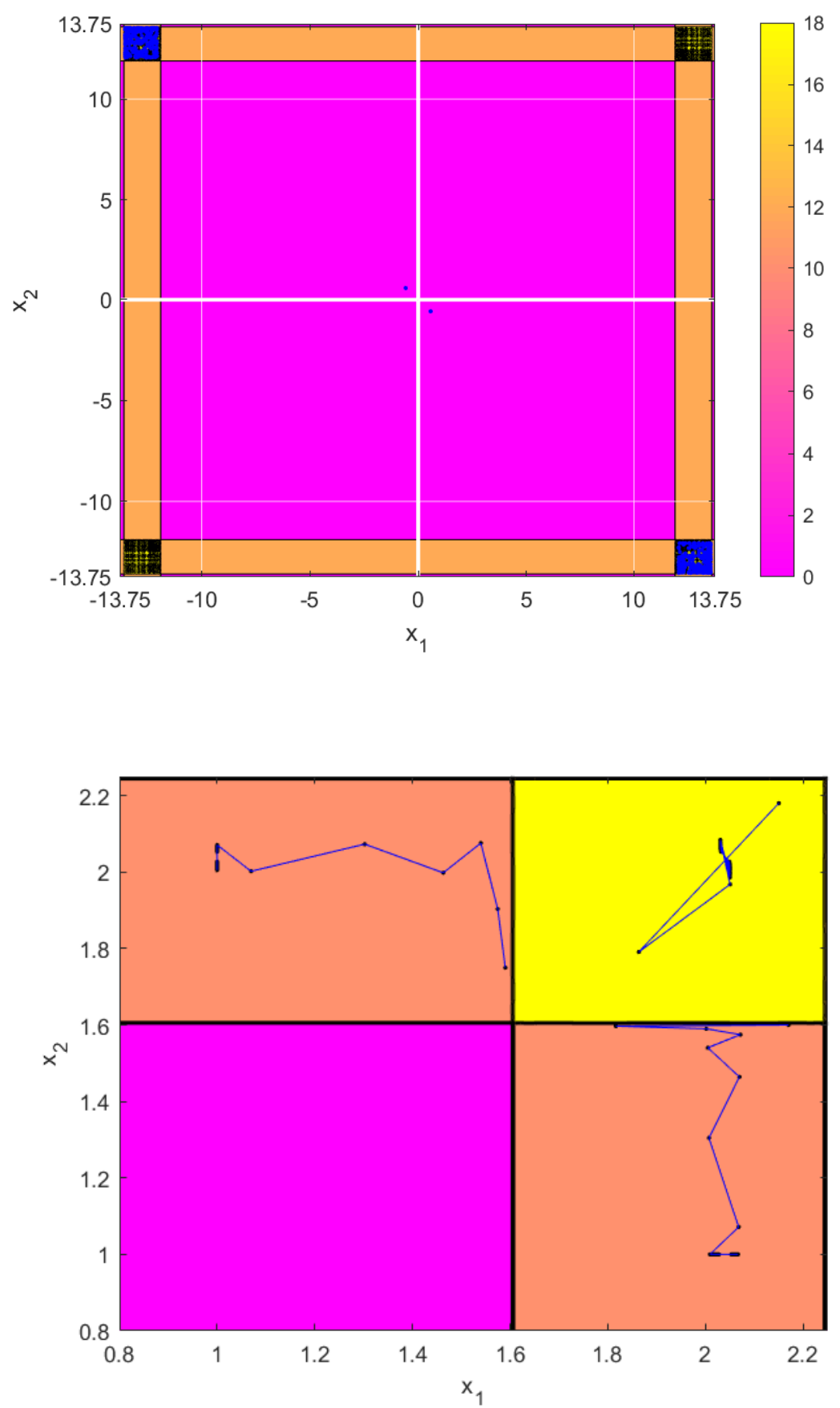